\title{
Quaternion Atomic Phase Magnification for 2D and 3D Motion
}

\author{
E. Ulises Moya-Sánchez ${ }^{1}$, Marcela Bonell ${ }^{1}$, Gustavo Torres ${ }^{1}$, \\ Eduardo Vázquez-Santacruz ${ }^{2}$ \\ 1 Universidad Autónoma de Guadalajara, Guadalajara, \\ Mexico \\ 2 Universidad Nacional Autónoma de México, Mexico City, \\ Mexico \\ eduardo.moya@edu.uag.mx,marcelabonell@gmail.com,gustavo.blanco@edu.uag.mx, \\ evazquezsa@conacyt.mx
}

\begin{abstract}
The phase computation is used in many applications of signal and image processing such as edge, line, symmetry detection, image analysis and recently to show small motion or color changes. Eulerian video processing allows to show small color changes and motions invisibles to the naked eye. Eulerian motion magnification, was modify using the phase and the Riesz pyramid in order to reduce the noise amplification. In this work we introduced a novel phase magnification approach based on a infinitely differentiable unique compact supported window, an atomic function. Another contribution of our work was apply our quaternion phase and Eulerian magnification to 3D motion, which has not been reported so far, we propose to use simple projections of conformal algebra in order to do the magnification at any plane of the 3D space. We compare the phase (Riesz Pyramids), with the atomic Riesz pyramid, in our comparation our atomic pyramid Riesz preserves more information than the phase Riesz approximation, besides our approach is slower by 2.5 times. Finally, we compare method and the linear magnification (Laplace Pyramid) in terms of the Signal to Noise Ratio (SNR). We found that the phase magnifications has better response to noise than the linear method.
\end{abstract}

Keywords: Quaternion atomic phase, phase magnification, atomic functions, and local phase.

\section{Introduction}

The phase concept is commonly used in many application areas of signal processing such as telecommunications, image processing, geophysics, hypercomplex analysis, and recently to show small motion or color changes, visual microphone and to create an interactive elastic image called visual vibrometry $[15,14,16,17,8,9]$. There are three different types of phases, the global or Fourier phase, the instantaneous phase and the local phase [7,12]. In this work, we propose a new technique for computing the local phase magnification in order to amplify motion by using a multiscale radial atomic function and $1 \mathrm{D}$ atomic function for computing the short-term and long-term temporal 
variation on $2 \mathrm{D}$ and $3 \mathrm{D}$. There are three main reasons for using the atomic functions. The atomic function have a compact support in space domain, the $n$-order derivative is easy to compute and we can compute the Hilbert and the Riesz transform by using the first derivative [6]. We present some applications in order to measure the breathe rate in $2 \mathrm{D}$ and $3 \mathrm{D}$ and the heart beat measurement. We use a RGB camera and RGBD (3D) sensors KinectI to get 2D and 3D sequences. According to our results, our phase magnification lost less information than the other Riesz approximation, however the computational time of our method response to noise than the linear method.

\section{Quaternion Algebra}

Quaternions $\mathscr{H}$ or $\mathscr{G}_{3,0,0}$ in the Geometric algebra $G A$ is correct framework to compute the local phase $[11,12,7]$. An element of $\mathscr{H}$ consists of a real element adding three imaginary elements $(i, j, k)$ :

$$
q=a+b i+c j+d k
$$

where $a, b, c, d \in \mathscr{R}$ and $i, j$ obey the relations $i^{2}=j^{2}=-1, i j=k$. The real part of $q$ is noted by $\operatorname{Re}(q)=a$ and the pure part is $P u(q)=b i+c j+d k$ [13]. $\mathscr{H}$ are geometrically inspired, due to the quaternions may be used to represent rotations (as bivector) in $\mathscr{R}^{3}$ and $\mathscr{R}^{4}$ and translations (as vector) in $\mathscr{R}^{3}$ [13]. GA allows to distinguish naturally objects and operations [12]. The imaginary components can be described in terms of the basis of $\mathscr{R}^{3}$ space, $i \rightarrow \mathrm{e}_{32}, j \rightarrow \mathrm{e}_{13}, k \rightarrow \mathrm{e}_{21}[12,13]$.

\section{Local Phase Information}

The Fourier or global phase is the most well known phase and denotes the angular phase of a signal in frequency domain [11]. However, when 2D the structural information is needed, the local phase can be computed [11,7]. The local phase, is used to know what is the phase at a certain position of the real signal [11,12]. The local phase means the computation of the phase restricted to a certain part or bandwidth of the real signal. The local phase is useful to separate the signal structure into impulses (even) and jumps (odd) $[15,14]$. Additionally, the phase information allows us to use the invariant or equivariant properties of the signal [12]. For instance, it has been shown that the phase has an invariant response to image brightness and it can also be invariant to the rotations $[12,14]$. For $2 D$ signals, the complex approach, Hilbert transform is not enough to compute the magnitude and local phase independently in any direction $[12,11]$. Then, the quaternionic analytic signal and the monogenic signal have been proposed $[11,12,15]$.

\subsection{Monogenic Signal}

The monogenic signal, was proposed by M. Felsberg and G. Sommer, and generalize the analytic signal to $n-\mathrm{D}$. The monogenic signal for $2 D$ signals is represented by [7]:

$$
f_{\mathbf{M}}(\mathbf{x})=f(\mathbf{x})+(i, j) f_{\mathbf{R}}(\mathbf{x})=f(\mathbf{x})+(i, j) f(\mathbf{x}) * \frac{\mathbf{x}}{2 \pi|\mathbf{x}|^{3}} .
$$


The magnitude of the signal is computed by $\left|f_{\mathbf{M}}(\mathbf{x})\right|=\sqrt{(i, j) f_{\mathbf{R}}^{2}+f(\mathbf{x})^{2}}$. Since the monogenic signal is constructed from the original signal and its Riesz transform, we can express the local phase $\phi$ and the local orientation $\theta$ as [12]:

$$
\begin{aligned}
& \phi=\arctan \left(\frac{\left|(i, j) f_{\mathbf{R})} * f(\mathbf{x})\right|}{f(\mathbf{x})}\right), \\
& \boldsymbol{\theta}=\arctan \left(\frac{j f_{\mathbf{R}} * f(\mathbf{x})}{i f_{\mathbf{R}} * f(\mathbf{x})}\right) .
\end{aligned}
$$

We use an special window, the radial atomic function, in order to compute the local phase, to a certain part or bandwidth of the real signal.

\section{Atomic Functions and Monogenic Signal}

In this work we use two atomic functions an $u p(x)$ for time convolution and a radial atomic function $\operatorname{Plop}(x, y)$ in order to compute the Riesz Transform in a multiscale approach.

The atomic functions $(A F)$ are compactly supported, infinitely differentiable solutions of differential functional equations with a shifted argument [18]:

$$
L f(x)=\lambda \sum_{k=1}^{M} c(k) f(a x-b(k)),|a|>1, b, c, \lambda \in N,
$$

where $L=\frac{d^{n}}{d x^{n}}+a_{1} \frac{d^{n-1}}{d x^{n-1}}+\ldots+a_{n}$ is a linear differential operator with constant coefficients.

The function $u p(x)$ has the following representation in terms of their Fourier transform as $[18,5]$ :

$$
\begin{aligned}
u p(x) & =\frac{1}{2 \pi} \int_{\mathscr{R}} \prod_{k=1}^{\infty} \frac{\sin \left(v 2^{-k}\right)}{v 2^{-k}} e^{i v x} d v \\
& =\frac{1}{2 \pi} \int_{\mathscr{R}} \hat{i p}(v) e^{i v x} d v
\end{aligned}
$$

A radial atomic function was mentioned in [6] as $u p\left(\sqrt{x^{2}+y^{2}}\right)$ (see Fig 1). However in [6], the function $\operatorname{Plop}(x, y)$ was defined as a radial infinite differentiable function with compact support (see Fig 1):

$$
\operatorname{Plop}(v, v)=\prod_{h=0}^{\infty} \sum_{k=0}^{\infty} \frac{\left[-\left(u^{2}+v^{2}\right)\right]^{k}}{3^{2 k(h+1)}[(k+1) !]^{2}},
$$

and is solution of the following functional-differentail equation:

$$
\nabla^{2} \operatorname{Plop}(x, y)=\lambda \int_{\partial S} \operatorname{Plop}\left[3\left(x-\xi_{1}\right), 3\left(y-\xi_{2}\right)\right] d s+\mu \operatorname{Plop}(3 x, 3 y),
$$

where $\nabla^{2}=\Delta=e_{1} \frac{\partial^{2}}{\partial x^{2}}+e_{2} \frac{\partial^{2}}{\partial y^{2}}, \xi_{1}^{2}+\xi_{2}^{2}=4 / 9, \mu=-4 \pi \lambda / 3$ and $\lambda=3^{5} / 4 \pi$. 

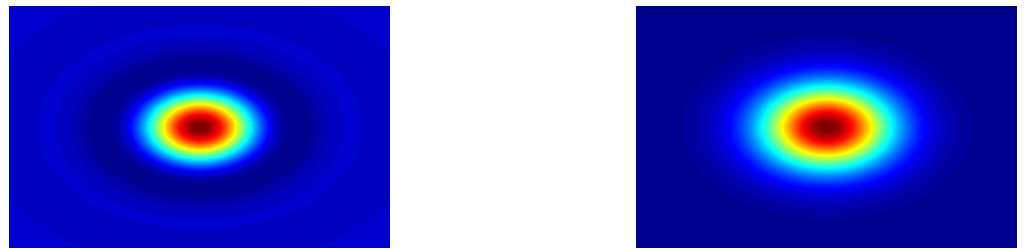

Fig. 1. Left: $\hat{u p}\left(\sqrt{v^{2}+v^{2}}\right)$; right: $P l \hat{o p}(v, v)$.

\subsection{Riesz Transform using Atomic Functions}

The Hilbert transform acts as an involution on the space of solutions of linear differentialfunctional equations solutions such as atomic functions [19]. The Riesz transform can be seen as a generalized Hilbert transform to $n$-dimensions [12]. A Riesz transform based on an atomic function was presented at [6] using this equation:

$$
f_{\mathbf{R}}(\mathbf{x})=f(\mathbf{x}) *\left(\nabla^{2} \operatorname{Plop}(\mathbf{x}) *-\frac{1}{2 \pi} \operatorname{sign}(|\mathbf{x}|) \log (|\mathbf{x}|)\right) .
$$

Atomic-Riesz pyramid coefficients consist of a real part and two imaginary parts with two Riesz transforms based on equation 10:

$$
f_{\mathbf{M}}(\mathbf{x})=f(\mathbf{x})+i f_{\mathbf{R}_{1}}(\mathbf{x})+j f_{\mathbf{R}_{2}}(\mathbf{x}),
$$

and this information is used to determine the local amplitude $A=\left\|f_{\mathbf{M}}\right\|$, local phase $\phi$ and local orientation $\theta$ :

$$
\begin{array}{r}
f_{\mathbf{M}}=A \cos (\phi)+i A \sin (\phi) \cos (\theta)+j A \sin (\phi) \sin (\theta), \\
\log \frac{f_{\mathbf{M}}}{\left\|f_{\mathbf{M}}\right\|}=i A \phi \cos (\theta)+j A \phi \sin (\theta) .
\end{array}
$$

Equation 13 uses a normalized quaternion, and is invariant to whether the local phase and orientation are $\phi$ and $\theta$ or the antipode $-\phi$ and $\theta+\pi$.

\section{$5 \quad$ Linear and Phase Magnification}

Eulerian video magnification (linear magnification), was introduced by F. Durand, W. Freeman et al $[1,8,9]$ is able to amplify small motions or color changes in videos. In their work, the temporal brightness changes in each frame sub-bands are amplified in order to amplify motions or color changes.

Figure 2 shows the Euler magnification approach on an image, by increasing temporal variation it is possible to increase spatial motion or color.

In order to do the implementation of the linear magnification Wadhwa et al. [8] use a Laplace pyramid in addition to temporal filter. The main problem of linear magnification is that noise power is amplified. 


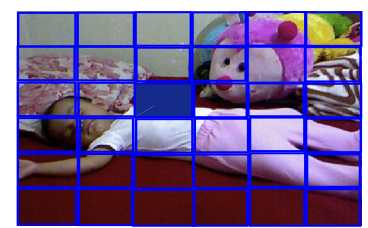

Fig. 2. Euler magnification approach on a frame, where the fluid par- ticles is tracked over time.

Several papers has demonstrated that the local phase using bandpass filtered can be used for local structure description and motion estimation. Also it has been shown the link between phase and motion and could be exploited in an Eulerian manner for motion magnification [14,20]. Additionally, the main problem of linear magnification were solved by amplifying temporal phase variations using a Riesz pyramid approximation $[1,10]$. In this work we propose use an unique atomic function to compute our Riesz transform aproximation and to compute some temporal filters in 1 dimension.

\subsection{Local Phase Magnification based on Atomic Functions}

As a summary, the first step to phase magnification is to express the image sequence in terms of the quaternion Riesz pyramid. The next step is to filter the local phase in a temporal way we have used an $u p(x)$. Then an amplify factor $(\alpha)$ was applied to the local phase(magnitude and local orientation are not affected), finally the Quaternion Riesz pyramid of the $n$ frames is reconstructed. This method is very time consuming due to we need 3 images ( or 3 pyramids) for each sequence pyramids instead of one pyramid a Laplacian approach. The local phase computation has an ambiguous sign, therefore a filter a sequence of unit quaternions can be used. According to $[8,9]$ the local orientation $\theta$ is supposed as constant over time at every pixel [9]. The equation 13 can be expressed in terms of $n$-frames:

$$
i A \phi_{n} \cos (\theta)+j A \phi_{n} \sin (\theta) .
$$

At every pixel, a temporal filtering $1 D$ on this quantity to isolate motions or changes of interest. In this case we propose using an $1 \mathrm{D}$ atomic function $u p(x)$. Spatial filtering can be achieved by weighted blur with Plop function on the $i$ and $j$ components:

$$
\text { iPlop }(x, y) A \phi_{n} \cos (\theta)+j \text { Plop }(x, y) A \phi_{n} \sin (\theta) .
$$

Motion amplify coefficients in the phase approach are in the same way as phase-shift a complex number. A quaternion expression is amplified by $\alpha$ factor such as

$$
A \cos (\alpha \phi)+i A \sin (\alpha \phi) \cos (\theta)+j A \sin (\alpha \phi) \sin (\theta) .
$$

When multiply $I_{M}$ this unit quaternion by the original coefficient $(\mathbf{x})+i f_{\mathbf{R}_{1}}(\mathbf{x})+$ $j f_{\mathbf{R}_{2}}(\mathbf{x})$ the real part can be expressed by [9]:

$$
I_{M}=A \cos (\alpha \phi)-A f_{\mathbf{R}_{1}} \sin (\alpha \phi) \cos (\theta)-A f_{\mathbf{R}_{2}} \sin (\alpha \phi) \sin (\theta) .
$$

In the results section we compare our approach with the method reported in [9] in terms of the Riesz pyramid reconstruction information and the time of computation. 


\section{3D Magnification}

The phase magnification was only defined by 2D projections. We use a RGBD sensor (Kinect I) in order to compute a 3D magnification. In this case we use a RGBD sensor in order to obtain the 3D information, therefore we have $(x, y)$ plane and the depth value is a query of the value coordinates, actually it is straightforward (see the distance such as brightness of the image) to apply the magnification in this image sequence.

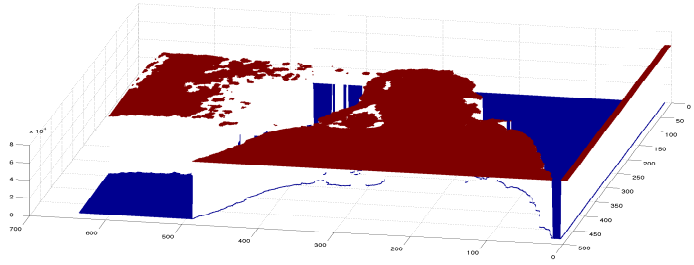

Fig. 3. Example of one 3D frame measure by kinect one.

However, using geometric algebra it is posible select any plane of magnification using the project or reject of the $3 \mathrm{D}$ points to another plane. The products of the Geometric Algebra has a geometric meaning and the dot product is related with the projection and the wedge product with the rejection.

Let $p_{l}=(x, y, z)$ the $3 \mathrm{D}$ points in our case measured by the RGBD sensor. It is posible to project the $3 \mathrm{D}$ point into anothers planes ( not only use $\pi_{x, y}=e_{1} \wedge e_{2}$ ) of interest using this equation in the conformal space:

$$
\begin{gathered}
P_{\text {project }}=\left(\pi \cdot p_{l}\right) \pi, \\
P_{\text {reject }}=\left(\pi \wedge p_{l}\right) \pi,
\end{gathered}
$$

where $\pi=e_{l} \wedge e_{m}$ is plane where the 3D points will be projected, $P_{\text {project }}$ are the 3D points projected in a new plane, in the case of the rejection points $P_{\text {reject }}$ will be on orthogonal plane. For instance if we want to project in the $\pi_{y, z}=e_{2} \wedge e_{3}$. A similar equation (using a dot product) can be used to project lines, planes, spheres among others objects. We belive that this is a good example about how use geometric algebra in low and mid level image processing.

\section{Results and Analysis}

\subsection{D Magnification}

The original gray image and its Region of Interest (ROI) in the chest of a baby sleeping is shown in the Fig. 4. In order to show the difference between the original sequence and the magnified sequence. We select this ROI due to is a very significant information 


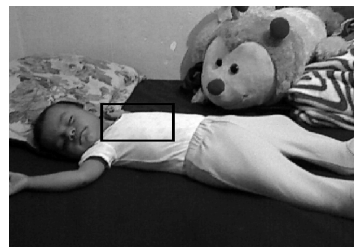

Fig. 4. ROI of the image sequence for 2D magnification.

about the baby. This application can be used to make visible or measure the breathe rate using an simple camera.

Phase magnifications using the Riesz pyramid are presented in the Fig. 5. From left to right: original ROI, phase amplification of ROI and difference of each frame. In order to see the evolution of the signal we show from top to down we show 5 frames at $t=0: 0.5: 2 \mathrm{sec}$. Its posible to see in the difference image how the edge
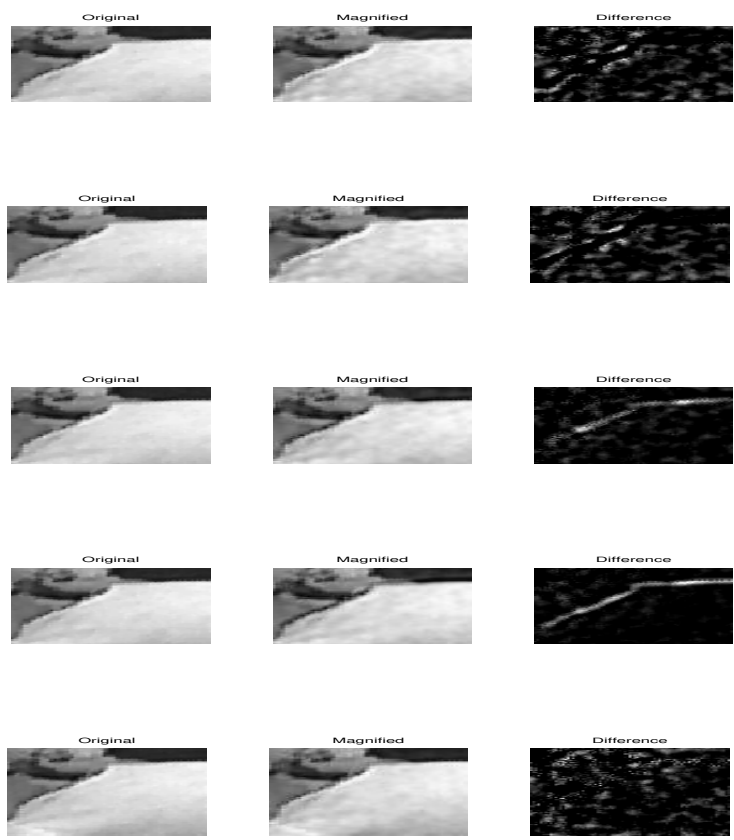

Fig. 5. From left to right: Original frame, linear magnification of a ROI of the image sequence and the difference between the original frame and its magnification.

An application of this technique is to measure the heart beat. In this case we present 


\section{E. Ulises Moya-Sánchez, Marcela Bonell, Gustavo Torres, Eduardo Vázquez-Santacruz}

the original image and its magnification Fig. 6 we can see in the subtraction of the images the white region related with the pulse rate. One of the important uses of sensing physiological signals of the human being, is the determination of stress levels or emotions in an automatic way and this two parameter can be used. We measure the heart beat using the maximum value and we get a $110 \mathrm{bpm}$.

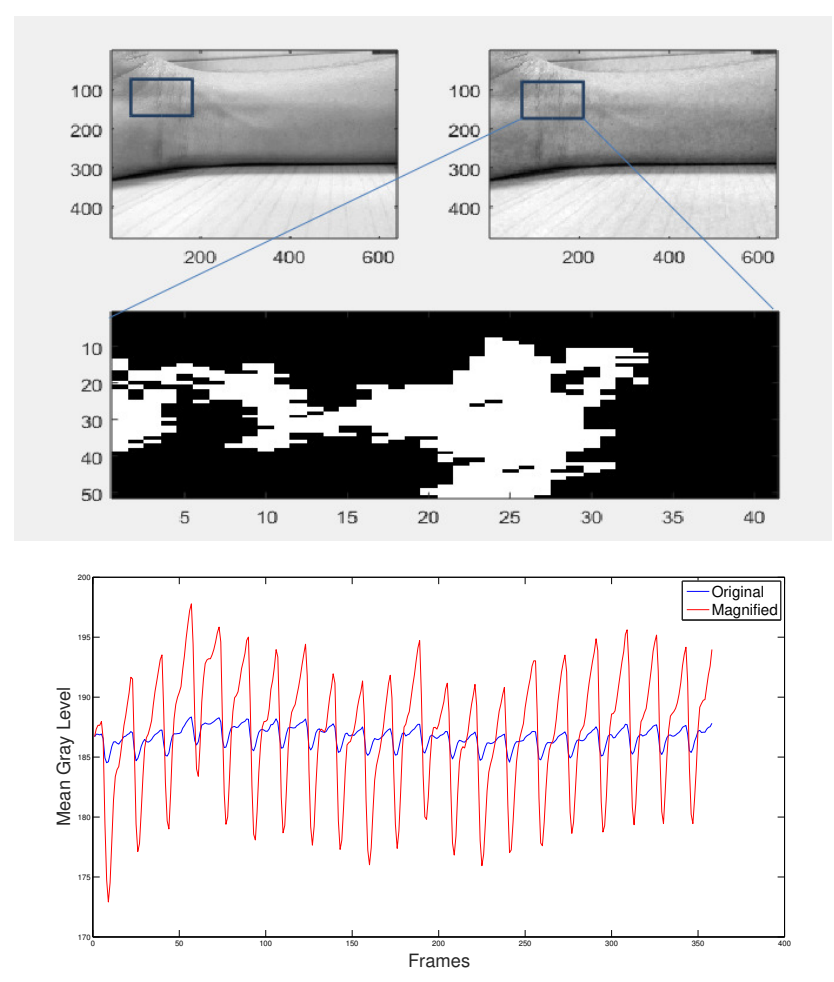

Fig. 6. Magnification of a ROI of the image sequence to get the heart beat.

\subsection{Riesz Pyramids Comparison}

In order to compare both approaches we compute de reconstruction (4 level in both cases) of a couple of images. The first image is a $256 \times 256$ with a white central point such as point spread function. In this case we show the original image, the reconstruction and finally an image subtraction. We measure de mean value of the difference image and the standard deviation. Our method obtain mean $=-2.6221 \times 10^{-24}$ $s t d v=1.0588 \times 10^{-22}$ in the difference image Fig. 7. In Riesz aproximation proposed by Wadwha was used fast Riesz computation using a 0D filter approximation based on five coefficients Chebyshev polynomials and the McClellan transform, the mean value 
of the difference was mean $=2.6622 \times 10^{-7}$ and a $s t d v=7.1790 \times 10^{-5}$. Its posible to see that our Riesz pyramid reconstruction presents less difference between the original.
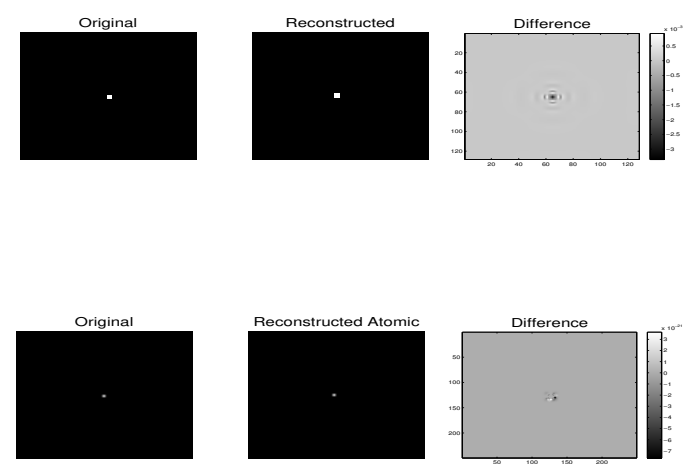

Fig. 7. From left to right: original image, the reconstruction of the image using the Riesz pyramid and finally the difference between the original and the reconstructed image. In the upper row a the result using Riesz pyramid proposed by Wadwha et all and in the down row our Riesz pyramid approximation using atomic functions.

\subsection{D Magnification}

One contribution of this work is amplify a 3D sensor image sequence. We use a Kinect, which provides a RGB-Depth images with $640 \times 480$ images at $30 \mathrm{fps}$. Due to IR Fig. 8 images has more noise than RGB images, therefore, we use a phase technique in order to improve a linear Eulerian magnification method in two aspects: it supports larger magnification and it has better noise performance.

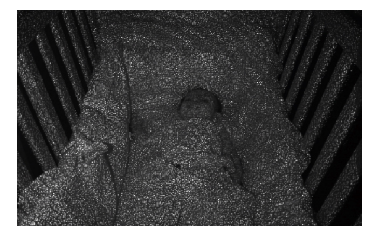

Fig. 8. IR image. It is possible to see the IR pattern has a lot of noise.

In this case we work with the original plane acquisition, which is by definition an $x, y$ plane where all 3D points are projected. We select the same chest baby in order to show the breath frequency. In Fig. 9 we present one frame and the selected region of interest used in the Fig. 10. We use the depth map from the Kinect, we present the 3D motion magnification Fig 10. 


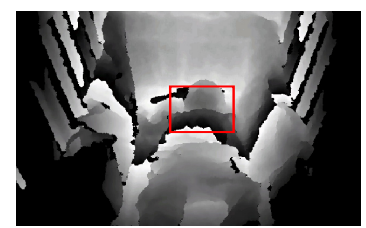

Fig. 9. Depth image. We select a ROI of 3D image using the Kinect.
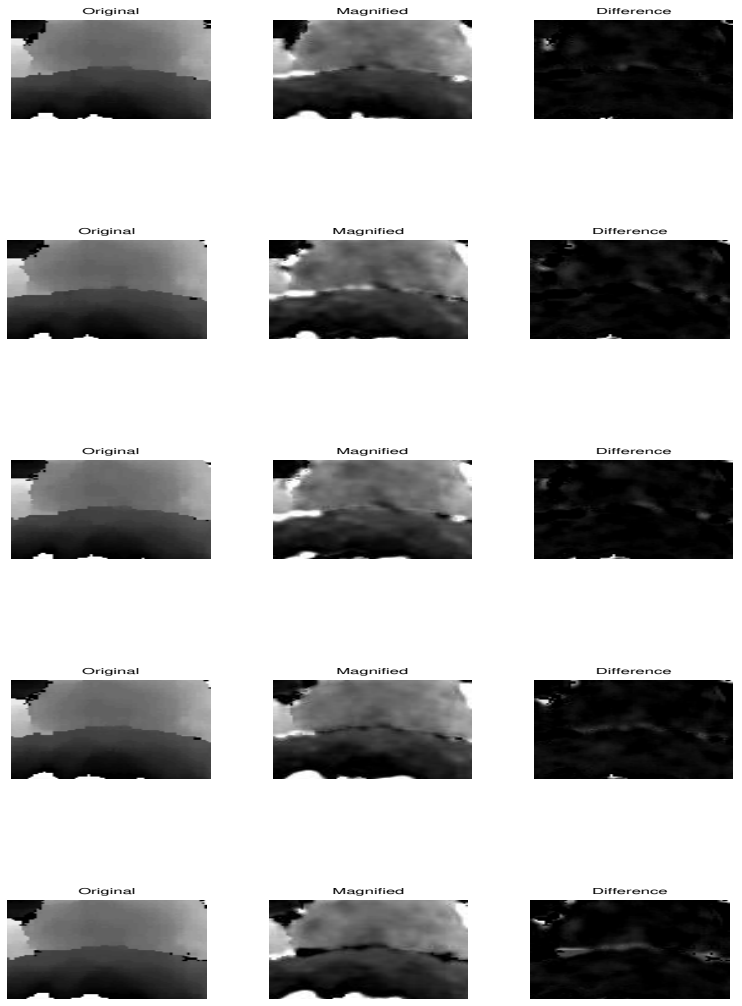

Fig. 10. Depth image phase magnification of the selected ROI. From left to right, original ROI, phase amplification of ROI and difference of each frame between the original ROI and the magnified frame. From top to down we show 5 frames at $t=0: 0.5: 2 s$.

In the selected ROI, we compute the Signal to Noise Ratio in terms of the mean value of the ROI (signal) and the standard deviation of the ROI (Noise) of the magnification areas. We can see that the linear magnification Fig. 11 has more variations, in contrast phase magnification Fig. 12 has less noise at the breath peaks. This results shows that it is posible to make a $3 \mathrm{D}$ phase magnification. In a future work we can 
show the 3D magnification in different planes using conformal geometry. This result has the same behavior that the reported by Wadwha et all. Where the linear magnification amplify the noise of the signal. We believe that our results could be used with another $3 \mathrm{D}$ sensors based on the projection and rejection equation it easy to project all the point to a plane of magnification. In a future work we hope to apply this 3D with another 3D objets such as lines, spheres among others.

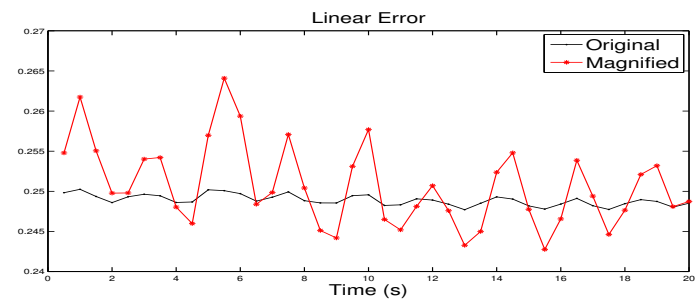

Fig. 11. Standard deviation of the selected ROI using a linear magnification.

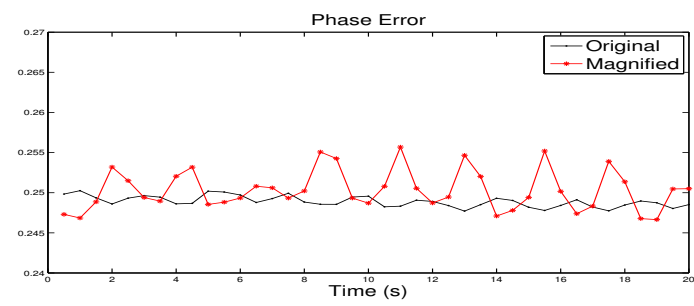

Fig. 12. Standard deviation of the selected ROI using a Phase magnification.

\section{Conclusions}

As a conclusion, we have presented a new local phase quaternionic method based on a unique compact supported window infinitely differentiable, the atomic function $u p(x)$. We compute a Riesz pyramid based on atomic functions and compare with the Wadhwa Riesz approximation, our method present less difference between the gray scale value of each image. However, the fast phase computation made by Wadwha takes 2.5 less time than our method. Additionally we do not see a significance difference in the final magnification. In our method we see in some levels an alliasyng effect at the high pass filtered images. Additionally we have presented a 3D magnification based on IR depth sensor KinectI in the front plane of the sensor. We present a projection equation in conformal geometry in order to compute another magnification in others projections. Due to IR images of both sensor have more noise than RGB images we 
use a phase technique to improve the linear Eulerian magnification method in two aspects: it supports larger magnification and it has better noise performance. Finally we compare, the Riesz Pyramids method and the linear magnification in terms of the Signal to noise ratio (SNR), we found that the phase magnifications has better response than the linear method. The phase motion magnification method has higher quality, however it is also more expensive for computing which is similar result previously reported. Also, considering another future application in forensic science, we can apply our tools in order to detect small changes in images about facial gestures or small changes by blood pressure, pulse and respiration in faces images given some questions put to person. In this sense, we will have a detector of small changes in people's faces images (2D or 3D models) to a set of questions, only using cameras and image processing.

Acknowledgment. Thanks to our research team and volunteers of our videos and we want to thank to CONACYT-SNI.

\section{References}

1. Rubinstein, M.: Analysis and Visualization of Temporal Variations in Video. PhD thesis, Massachusetts Institute of Technology (2014)

2. Davis, A., Bouman, K. L., Chen, J. G., Rubinstein, M., Durand, F., Freeman, W.T.: Visual vibrometry: Estimating material properties from small motions in video. In: IEEE Conference on Computer Vision and Pattern Recognition (CVPR) (2015)

3. Moya-Sánchez, E.U., Bonell, M.: Quaternion Atomic Phase Magnification for 3D Motion. In: Early Proceedings of the AGACSE Conference (2015)

4. Moya-Sánchez, E.U., Bayro-Corrochano, E.: Quaternion and Clifford Fourier Transforms and Wavelets. Ch 4: Quaternionic Local Phase for Low-level Image Processing Using Atomic Functions Trends in Mathematics, pp. 57-83, Springer (2013)

5. Bayro-Corrochano, E., Ulises Moya-Sánchez, E.: Ch 8: Quaternion Atomic Function for Image Processing, Leo Dorts Joan Lasenby. Guide to Clifford Algebras in Practice, Spinger (2011)

6. Moya-Sánchez, E.U., Bayro-Corrochano, E.: Hilbert and Riesz transforms using atomic function for quaternionic phase computation. Adv. Appl. Clifford Algebr. 23, No. 4, pp. 929-949 (2013)

7. Felsberg, M., Sommer, G.: The monogenic signal. IEEE Trans. Signal Process., 49(12), pp. 3136-3144 (2001)

8. Wadhwa, N., Rubinstein, M., Durand, F., Freeman, W.T.: Riesz Pyramids for Fast PhaseBased Video Magnification. In: IEEE International Conference on Computational Photography (ICCP) (2014)

9. Wadhwa, N., Rubinstein, M., Durand, F., Freeman, W.T.: Quaternionic Representation of the Riesz Pyramid for Video Magnification, http://people.csail.mit.edu/nwadhwa/rieszpyramid

10. Wadhwa, N., Rubinstein, M., Durand, F., Freeman, W.T.: Pseudocode for Riesz Pyramids for Fast Phase-Based Video Magnification Tecnical Report (2014)

11. Bülow, T.: Hypercomplex spectral signal representations for the processing and analysis of images. PhD thesis, Christian- Albert, Kiel University, Kiel, Germany (1999)

12. Felsberg, M.: Low-level image processing with the structure multivector. $\mathrm{PhD}$ thesis, Christian- Albert, Kiel University, Kiel, Germany (2002) 
13. Lounesto, P.: Clifford Algebras and Spinors. Cambridge University Press (2001)

14. Kovesi, P.: Invariant measures of image features from phase information. $\mathrm{PhD}$ thesis, University of Western Australia (1996)

15. Lennart, W.: Local feature detection by higher order Riesz transforms on images. Master's thesis, Christian- Albert, Kiel University, Kiel, Germany (2008)

16. Ell, T.A., Sangwine, S.J.: Hypercomplex Fourier Transforms of Color Images. IEEE Trans Image Proc, 16(1), pp. 22-35 (2007)

17. Hitzer, E.M.S.: Quaternion Fourier Transform on Quaternion fields and Generalizations. J Adv Appl Clifford Alg, 17(3), pp. 497-517 (2007)

18. Kolodyazhnya, V.M., Rvachev, V.A.: Cybernetics and Systems Analysis, 43 (2007)

19. Weiss, J.: The Hilbert transform of wavelets are wavelets. Technical Report, Applied Mathematics Group (1995)

20. Bayro Corrochano, E.: The theory and use of the quaternion wavelet transform. Journal of Mathematical Imaging and Vision, 24, pp. 19-35 (2006) 